\title{
Exploring antioxidant reactivity and molecular structure of phenols by means of two coupled assays using fluorescence probe (2,3-diazabicyclo[2.2.2] oct-2-ene, DBO) and free radical (2,2-diphenyl-1-picrylhydrazyl, DPPH')
}

\author{
MERCEDES BECERRA-HERRERA ${ }^{\mathrm{a}, \mathrm{b}, *}$, ANA SAYAGO $^{\mathrm{a}}$ and RAFAEL BELTRÁN ${ }^{\mathrm{a}}$ \\ ${ }^{a}$ Department of Chemistry and Material Sciences, Area of Analytical Chemistry, Faculty of Experimental \\ Sciences, University of Huelva, Agrifood Campus of International Excellence, ceiA3. Avd. Tres de Marzo S/N, \\ 21007 Huelva, Spain \\ ${ }^{b}$ Department of Inorganic and Analytical Chemistry, Faculty of Chemical and Pharmaceutical Sciences, \\ University of Chile, P.O. Box 233, Santiago, Chile \\ E-mail: mercedes.becher@gmail.com; mercedes.becerra@dqcm.uhu.es
}

MS received 14 March 2016; revised 26 May 2017; accepted 18 June 2017; published online 11 July 2017

\begin{abstract}
Phenolic compounds can be considered as the most important bioactive compounds in Mediterranean diet. However, many of the complex connections between phenols antioxidant reactivity and their molecular structure remain unsolved. To shine light on these issues, the antioxidant reactivity of 15 relevant phenolic compounds was studied. Two different analytical approaches were combined: (a) the well-established 2,2diphenyl-1-picrylhydrazyl (DPPH') stable free radical assay, and (b) the 2,3-diazabicyclo[2.2.2] oct-2-ene (DBO) fluorescent probe assay. The results obtained confirm how certain structural features (i.e., hydroxyl and methoxy groups, alkyl and alkenyl chains) play a critical role in the singular antioxidant response exhibited by each phenolic compound. This knowledge provides decisive information to select a specific phenolic compound as an antioxidant additive or for the chemical design of new antioxidants.
\end{abstract}

Keywords. Phenolic compounds; DPPH; 2,3-diazabicyclo[2.2.2]oct-2-ene (DBO); antioxidant activitystructure relationship.

\section{Introduction}

Phenolic compounds are some of the most important bioactive compounds in human diet. Throughout the years, more than 8000 phenolic compounds have been identified within the following generic chemical groups: phenolic acids, phenolic alcohols, flavonoids, stilbenes and lignans. ${ }^{1,2}$ The present study will focus its effort on the most relevant phenolic compounds in olive oil and wine. ${ }^{3,4}$ These food products have shown countless benefits for human health, ${ }^{4}$ and can be fairly considered to be essential ingredients of the Mediterranean diet.

Phenols are potent antioxidants ( $\mathrm{ArOH})$ due to their high ability to scavenge free radicals $\left(\mathrm{R}^{\mathrm{P}}\right){ }^{5}$ They prevent food degradation and some human diseases like arthritis, artherosclerosis, ${ }^{6}$ several types of cancer, coronary heart disease, ${ }^{7}$ and provide protection against inflam-

\footnotetext{
*For correspondence
}

mation. ${ }^{8,9}$ The main structural feature responsible for the antioxidant reactivity of phenolic compounds is the hydroxyl group. This group is able to donate hydrogen atoms to a free radical, stopping the propagation chain during the oxidation process, and thereby inhibiting or slowing down the whole oxidation process. Peroxyl radicals (ROO) are the most common chain carrying radicals. Typically, the hydrogen transferred from a phenolic compound to the peroxyl radical forms a hydroperoxide $(\mathrm{ROOH})$, and a much less reactive radical $(\mathrm{ArO})$ derived from the antioxidant. It is also well known that the presence of a second hydroxyl group at the ortho-position can increase the rate at which hydrogen is transferred to peroxyl radicals. ${ }^{10}$ In addition, the alkyl chain that connects the phenolic ring and the carboxylic or alcohol group that contains some phenolic compounds may be important to stabilize the phenoxy radical formed (ArO $).{ }^{11}$ 
The last two decades have seen a vibrant proliferation of multiple assays to measure phenolic antioxidant reactivity in the field of Food Chemistry. Among others, it is imperative to acknowledge the following spectroscopic techniques: electron spin resonance (ESR), ${ }^{12,13}$ stable 2,2-diphenyl-1-picrylhydrazyl radical (DPPH'), ${ }^{12-15}$ 2,2'-azino-di[3-ethylbenzthiazoline sulfonate] radical cation (ABTS+), ${ }^{16}$ oxygen radical absorption capacity (ORAC), ${ }^{17} \beta$-carotene-linoleate model system ${ }^{14}$ and ferric reducing antioxidant capacity (FRAP). ${ }^{17}$

The DPPH radical assay can be considered the prevailing method to study antioxidant reactivity of phenols. This versatile and practical assay is mainly due to following factors: 1) monomeric behavior in solution, 2) stability in aerobic conditions, 3) commercial availability, 4) intense initial color in solution and 5) strong color change after hydrogen scavenging. Besides, the reaction between phenols $(\mathrm{ArOH})$ and $\mathrm{DPPH}$ (reaction 1 ) or phenols and peroxyl radicals (ROO, reaction 2) can be considered as equivalents.

$$
\begin{aligned}
& \mathrm{ArOH}+\mathrm{DPPH} \rightleftarrows \mathrm{ArO}+\mathrm{DPPH}-\mathrm{H} \quad \text { (reaction1) } \\
& \mathrm{ArOH}+\mathrm{ROO} \rightleftarrows \mathrm{ArO}+\mathrm{ROOH} \quad \text { (reaction2) }
\end{aligned}
$$

Nevertheless, some minor disadvantages need to be considered, among others: 1) the long period of time until the reaction is completed (typically in the range of 15 to $30 \mathrm{~min}), 2$ ) the presence of spectral interferences, ${ }^{18}$ and 3 ) the concomitant limitations of the use of conventional spectrophotometers.

In order to complement the blanket approach on the study of phenols antioxidant reactivity offered by the DPPH assay and to avoid the mentioned limitations of the spectrophotometric methods, a methodology based on fluorescence quenching was applied. To this end, the fluorescence probe, $n, \pi^{*}$ singlet-excited state of 2,3diazabicyclo[2.2.2] oct-2-ene (DBO) was chosen. This specific fluorescence probe has shown great efficiency on the study of antioxidant reactivity of phenols. ${ }^{19-23}$ It is important to emphasize that fluorescence quenching can be resolved with high sensitivity towards the mechanism and action of antioxidants. Additionally, it is highly selective, easy to apply and less time consuming than the DPPH assay.

Comparing both probes, the steric effect present in the DPPH assay is absent in the DBO assay, because the groups around the reactive site of DBO $\left(\mathrm{N}^{*}\right)$ are much less bulky than those of DPPH $\left(\mathrm{N}^{\prime}\right) .{ }^{24}$ Hence, the coupled use of DPPH and DBO assays is shown in the present study as a routine, robust and comprehensive analytical method to study antioxidant reactivity of phenols. By means of this combined methodology, the antioxidant reactivity of 15 selected phenols (typically present in olive oil and wine) is explored as well as the close relationship between molecular structure of each phenol and its antioxidant response.

\section{Experimental}

\subsection{Materials}

The 15 selected phenolic compounds for this study are shown in Fig. 1.

Commercially available compounds were used as received. Gallic acid (99\%), protocatechuic acid (97\%), sinapic acid (98\%), and syringic acid (98\%) were purchased from Aldrich ${ }^{\circledR}$ (Darmstadt, Germany). Vanillic acid (97\%), tyrosol (97\%), p-hydroxyphenylacetic acid (100\%), caffeic acid (98\%), ferulic acid (99\%), cinnamic acid (99\%), $p$-hydroxybenzoic acid

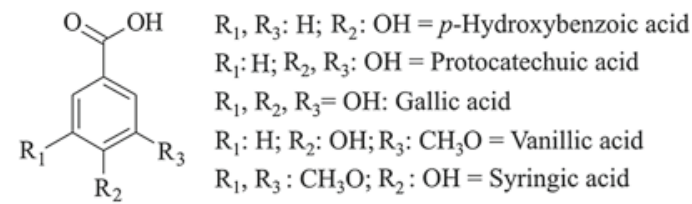

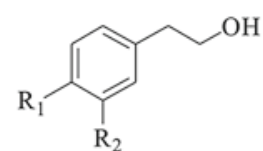

$\mathrm{R}_{1}: \mathrm{OH} ; \mathrm{R}_{2}: \mathrm{H}=$ Tyrosol

$\mathrm{R}_{1}, \mathrm{R}_{2}: \mathrm{OH}=$ Hydroxytyrosol

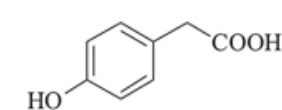

p-Hydroxyphenylacetic acid
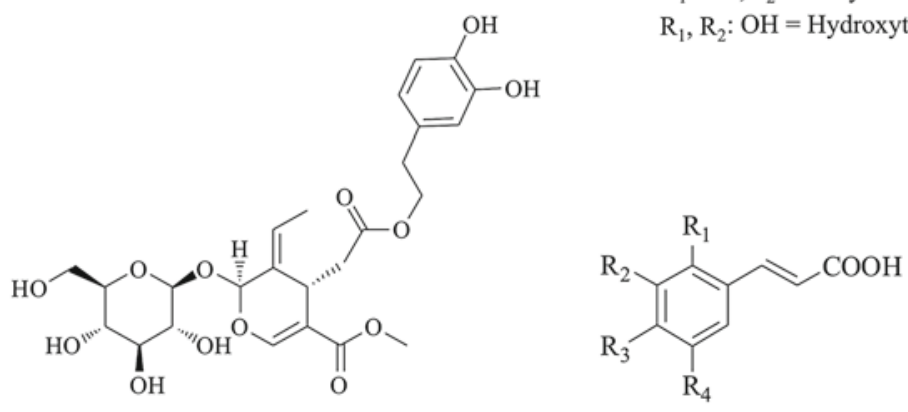

$\mathrm{R}_{1}, \mathrm{R}_{2}, \mathrm{R}_{3}, \mathrm{R}_{4}: \mathrm{H}=$ Cinnamic acid

$\mathrm{R}_{1}: \mathrm{OH} ; \mathrm{R}_{2}, \mathrm{R}_{3}, \mathrm{R}_{4}: \mathrm{H}=o$-Coumaric acid

$\mathrm{R}_{1}, \mathrm{R}_{2}, \mathrm{R}_{4}: \mathrm{H} ; \mathrm{R}_{3}: \mathrm{OH}=p$-Coumaric acid

$\mathrm{R}_{1}, \mathrm{R}_{4}: \mathrm{H} ; \mathrm{R}_{2}, \mathrm{R}_{3}: \mathrm{OH}=$ Caffeic acid

$\mathrm{R}_{1}, \mathrm{R}_{4}: \mathrm{H} ; \mathrm{R}_{2}: \mathrm{CH}_{3} \mathrm{O} ; \mathrm{R}_{3}: \mathrm{OH}=$ Ferulic acid

$\mathrm{R}_{1}: \mathrm{H} ; \mathrm{R}_{3}: \mathrm{OH} ; \mathrm{R}_{2}, \mathrm{R}_{4}: \mathrm{CH}_{3} \mathrm{O}=$ Sinapic acid

Oleuropein

Figure 1. Phenolic compounds structures. 
(99\%), $p$-coumaric acid (98\%) and $o$-coumaric acid $(97 \%)$ were purchased from Fluka ${ }^{\circledR}$ (Darmstadt, Germany). Oleuropein $(99 \%)$ was acquired from Extrasynthèse ${ }^{\circledR}$. Hydroxytyrosol was synthesized at the Organic and Pharmaceutic Chemistry Department, Faculty of Pharmacy (Seville, Spain) according following a literature procedure. ${ }^{25}$

DPPH (2,2-diphenyl-1-picrylhydrazyl, 99\%) was purchased from Fluka ${ }^{\circledR}$ (Darmstadt, Germany) whereas DBO fluorophore was synthesized at the Jacobs University of Bremen (Germany), according to the procedure proposed by Askani. ${ }^{26}$ Methanol was of spectrophotometric grade $\left(99.9 \%\right.$, Panreac ${ }^{\circledR}$, Barcelona, Spain). The ultrapure water was obtained from an in-house Milli-Q ${ }^{\circledR}$ Type I purification system $(18.2 \mathrm{M} \Omega \cdot \mathrm{cm}$ and TOC $<10 \mathrm{ppb})$.

An UV-Vis spectrophotometer (Termo Spectronic Helios$\gamma^{\circledR}$ V4.60, Cambridge, UK) was used for DPPH assay. A Cary Eclipse ${ }^{\circledR}$ spectrofluorimeter from Varian ${ }^{\circledR}$ (Santa Clara, United States) was used for the DBO fluorescence quenching studies. All measurements were performed at ambient temperature and in aerated solutions.

The results of the DPPH assay were processed using $\mathrm{JMP}^{\circledR}$ statistical software by means of a Principal Component Analysis (PCA), a multivariate statistical technique that allows a large set of data to be simplified and reduced into a smaller number of orthogonal factors (with magnitude and accuracy) to facilitate interpretation by visualizing the correlations that exist between the original variables. ${ }^{27}$

\subsection{DPPH radical scavenging assay}

The DPPH radical scavenging capacity of each phenolic compound was determined according to well-established and widespread method. ${ }^{28-30}$ The DPPH radical exhibits an absorption maximum at $515 \mathrm{~nm}$ that progressively disappears if the DPPH is reduced by an antioxidant compound. The DPPH assay is typically performed by dissolving both the DPPH radical and the antioxidant compounds in pure methanol or ethanol. ${ }^{30}$ Additionally, the present study explores the alternative use of water/methanol solvent to facilitate a comparison between the results obtained by the DPPH assay and by some recent HPLC-DAD and UHPLC-ESIMS/MS based methodologies. ${ }^{31,32}$ A fresh DPPH solution in pure methanol $(0.06 \mathrm{mM})$ was prepared daily during the study, whereas the different antioxidants $(0.5 \mathrm{mM})$, dissolved in pure methanol and in a water/methanol mixture (v/v, 76/24), were generated weekly. The absorbance was measured using a quartz cuvette (1 cm optical path length). Methanol was used as a blank reference. $1 \mathrm{~mL}$ of antioxidant stock solution was added to $2 \mathrm{~mL}$ of DPPH solution and the absorbance at $515 \mathrm{~nm}$ was monitored each second until it reached a steady state $(90 \mathrm{~min})$ at $20^{\circ} \mathrm{C}$, using a UV-Vis spectrophotometer.

Previous studies have shown the need to correct the intrinsic DPPH bleaching (in the absence of any phenolic compound) if the reaction is monitored for a long period of time. $^{33}$ The use of a bleaching correction factor is essential to avoid a possible overestimation of the antioxidant reactivity. This bleaching effect was cautiously controlled during the present study and it was observed to be negligible for the duration time of the experiments (90 minutes). However, two additional correction factors were applied. Firstly, the addition of the phenolic compound dissolved in $1 \mathrm{~mL}$ of methanol or water/methanol mixture $(\mathrm{v} / \mathrm{v}, 76 / 24)$ dilutes the $2 \mathrm{~mL}$ of original DPPH solution, subsequently decreasing the initial color of the DPPH radical. To overcome this dilution effect, the following correction was applied:

$A_{t 0}^{c}=A_{t 0} \times 0.666( \pm 0.005)$

where, $A_{t 0}^{c}$ is the corrected initial DPPH absorbance (time = 0 ), $A_{t 0}$ is the initial DPPH absorbance (without antioxidant compound and $\mathrm{t}=0$ ) and $0.666 \pm 0.005$ is the dilution factor applied. This factor is the mean and standard deviation after measuring the dilution factor ten times $(n=10)$ in a blank solution which perfectly matched the expected linear response of a 1:2 stoichiometry.

Secondly, the complete reduction of the DPPH radical (induced by the antioxidants compounds) does not lead to a colorless and transparent solution. Instead, a soft yellowish final color appears when the DPPH' scavenging capacity is exhausted. This "residual color" induces an underestimation of the phenols antioxidant reactivity. A residual color factor in the range of 0.043 to 0.046 was estimated using the results of the present study (Figure S1) and the following correction of the results was proposed:

$A_{t}^{c}=A_{t}-0.044( \pm 0.002)$

where, $A_{t}^{c}$ is the corrected absorbance of the DPPH after antioxidant addition at time $t, A_{t}$ is the absorbance of the $\mathrm{DPPH}^{\circ}$ after antioxidant addition at time $t$, and $0.045 \pm 0.002$ is the mean and standard deviation of the residual color $(\mathrm{n}=$ 16).

The remaining DPPH in percentage was calculated using the following equation:

Remaining DPPH $(\%)=\left(A_{t}^{c} / A_{t 0}^{c}\right) \times 100$

where, $A_{t 0}^{c}$ is the corrected absorbance of DPPH at 0 min and $A_{t}^{c}$ is the corrected absorbance at each time interval. This equation was selected as the most appropriate one to calculate the remaining DPPH in percentage taking into consideration the previous corrections shown in eqs. 1 and 2. With the purpose to ensure the reliability of this methodology, these analyses were carried out in triplicate.

\subsection{DBO fluorescence quenching}

This assay is routinely performed dissolving the DBO fluorophore in pure water. ${ }^{23}$ However, following the reasoning exposed for the DPPH assay, it was also decided to investigate the feasibility of an alternative water/methanol solvent (v/v, 76/24). It is important to remark that the solubility of phenols in pure water is poor ( 8 of the 15 phenols studied are insoluble in pure water), whereas their solubility increased when a water/methanol solvent (v/v, 76/24) was used (only 4 of the 15 phenols of this study remained insoluble). The DBO concentration was matched to an absorbance of 0.05 at 364 

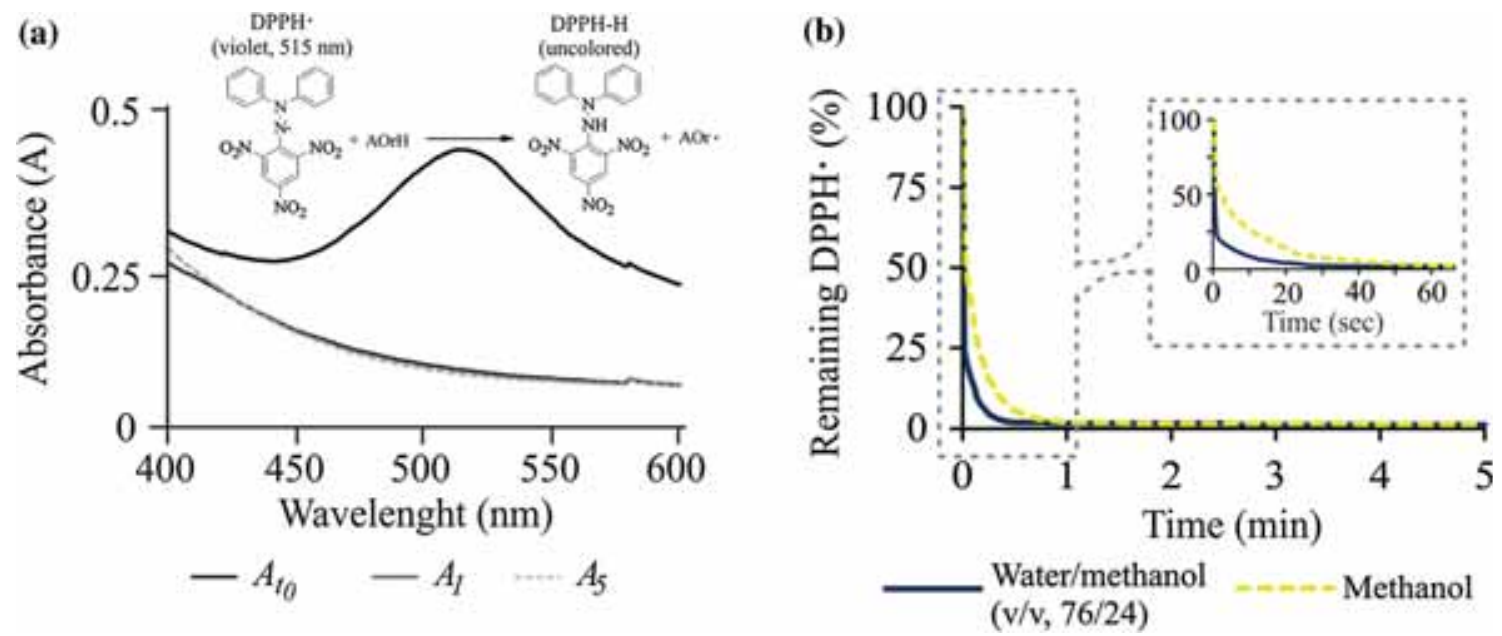

Figure 2. (a) Representative example of the raw absorbance spectra of DPPH obtained in the absence of antioxidant $\left(A_{0}\right)$ and at $1\left(A_{1}\right)$ and $5\left(A_{5}\right)$ minutes after the antioxidant (gallic acid, $\left.0.5 \mathrm{mM}\right)$ addition. The inset corresponds to DPPH structure in the absence of antioxidant and after its addition. (b) Illustrative example of the remaining DPPH $(\%)$ evolution with time (for $5 \mathrm{~min}$ ) after the antioxidant addition (gallic acid). Inset shows the remaining DPPH $(\%)$ evolution on the first minute $(60 \mathrm{~s})$ after the antioxidant addition. The results using methanol or water/methanol (v/v, 76/24) solvents are compared.

$\mathrm{nm}\left(\lambda_{\max }\right)$. In order to obtain the quenching for each antioxidant, $50 \mu \mathrm{L}$ aliquots of antioxidant stock solutions $(0.04 \mathrm{M})$ were added to $3 \mathrm{~mL}$ of DBO solution. The fluorescence spectrum was measured at an excitation wavelength of $364 \mathrm{~nm}$. The fluorescence quenching was monitored at the maximum of the DBO emission band (420 nm in water and $417 \mathrm{~nm}$ in water/methanol mixture (v/v, 76/24)). The data were plotted according to the Stern-Volmer equation:

$I_{0} / I=1+k_{q} \tau_{0}[\mathrm{ArOH}]$

Where, $I_{0}$ is the DBO intensity in the absence of antioxidant, $I$ is the intensity in the presence of antioxidant, $k_{q}\left(\mathrm{M}^{-1} \mathrm{~s}^{-1}\right)$ is the quenching rate constant, $\tau_{0}$ is the DBO lifetime in the absence of antioxidant, and $[\mathrm{ArOH}]$ is the antioxidant concentration.

The slope divided by the lifetime $\tau_{0}$ (in the absence of antioxidant quencher) afforded the bimolecular quenching rate constant $\left(k_{q}\right)$. As lifetime $\tau_{0}$ we used $325 \mathrm{~ns}$ for aerated water ${ }^{34}$ and $75.3 \mathrm{~ns}$ (measured by time-correlated-single photon-counting with an apparatus from Edinburgh Instruments) for the water/methanol mixture (v/v, 76/24).

\section{Results and Discussion}

\subsection{Radical scavenging reactivity}

The possibility of using a water/methanol mixture (v/v, $76 / 24$ ) to dissolve the DPPH radical was ruled out after several trials where the final solution was unstable and showed signs of incomplete DPPH dissolution. Therefore, it was decided to work always with DPPH dissolved in pure methanol. For each of the 15 phenolic compounds dissolved in methanol and methanol/water, the remaining DPPH (\%) was plotted as a function of time (data not shown). In general terms, in Figure $2 \mathrm{a}$, the changes of the DPPH absorption spectra obtained in the absence of antioxidant (read as $A_{0}$ at $\left.\lambda_{\max }\right)$ and its evolution after $1\left(\mathrm{read}\right.$ as $\left.A_{1}\right)$ and 5 (read as $A_{5}$ ) minutes of the antioxidant (gallic acid dissolved inwater/methanol; v/v, 76/24) addition are shown.

The initial violet color $(\lambda=515 \mathrm{~nm})$ is bleached due to the consumption of the DPPH, with the formation of colored products (derived from ArO'), less strongly absorbing than DPPH. It should be emphasized that the 'grow-in' of $\mathrm{ArO}$-derived products will not influence the measured kinetics of the $\mathrm{ArOH}+\mathrm{DPPH}$ reaction; i.e., there is no actual "interference". ${ }^{35}$

For the sake of simplicity and because of the similar response observed for the other studied phenols, it was decided to use gallic acid as a representative example of the general phenolic behavior. Figure $2 b$ shows a comparison between the time evolution of the remaining DPPH (\%) for the antioxidant (gallic acid) dissolved in pure methanol or in a water/methanol mixture $(\mathrm{v} / \mathrm{v}$, 76/24). As can be observed, the two DPPH consumption curves (using water/methanol mixture (v/v, 76/24) or pure methanol used to dissolve the phenolic compound) show great resemblance. However, a slightly faster reaction is noted if water/methanol solvent is used (Figure 2a). This kinetic behavior was consistently observed for all the phenolic compounds of this study, except for $p$-coumaric acid and $p$-hydroxyphenylacetic acid (data not shown). These observations support the 

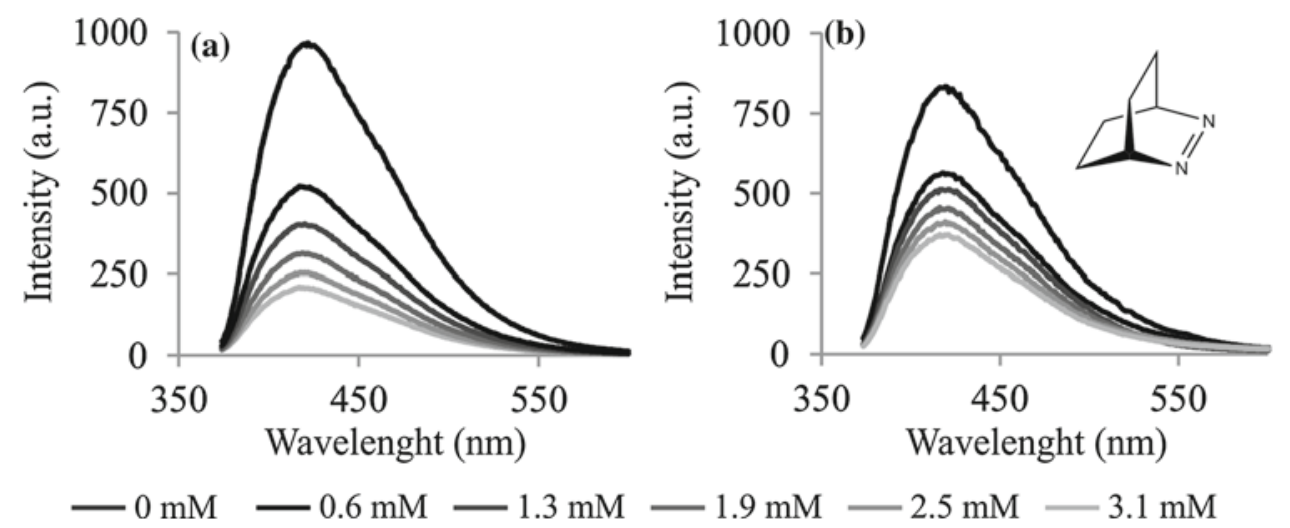

Figure 3. Evolution of the DBO fluorescence spectra obtained in (a) water and (b) water/methanol (v/v, 76/24) after adding varying amounts of gallic acid $(0,0.65,1.29$, 1.90 , and $2.5 \mathrm{mM}$, from top to bottom). Inset in Figure $3 \mathrm{~b}$ shows the structure of DBO.

use of either a pure methanol or water/methanol solvent to dissolve the phenolic compounds to be studied in a DPPH assay. For the sake of clarity and for an optimal comparison with other analytical techniques studying phenols, ${ }^{31,32}$ the discussion of the results of DPPH assay will be limited to the water/methanol solvent (v/v, 76/24).

\subsection{Fluorescence quenching of $\mathrm{DBO}$}

Upon excitation at $364 \mathrm{~nm}$, the typical fluorescence spectrum of DBO shows a maximum at $420 \mathrm{~nm}$ in water (Figure $3 \mathrm{a}$ ) and at $417 \mathrm{~nm}$ in water/methanol (Figure $3 \mathrm{~b}$ ). If an antioxidant (e.g., $p$-coumaric acid) is added, at increasing concentrations, a progressive decrease of the fluorescence intensity occurs (Figure 3).

Using the same initial concentration of DBO in both assays, the water/methanol mixture led to a lower fluorescence intensity of the fluorophore alone than observed for water as solvent. Notwithstanding, the fluorescence quenching assay using pure water solvent (Figure 3a) is more pronounced than the one using water/methanol solvent (Figure 3b). The amount of quenching, (quantified by experimental fluorescence intensity in the absence $\left(I_{0}\right)$ and presence $(I)$ of antioxidants), should be proportional to the concentration of the antioxidant and its reaction rate $\left(k_{q}\right)$ according to the Stern-Volmer expression. Accordingly, a plot of $I_{0} / I$ versus $[\mathrm{ArOH}]$ should yield a straight line with a slope equal to $k_{q} \tau_{0}$. Such a plot, known as a Stern-Volmer plot, was generated for each phenol in the present study, and obtained linear correlations for all of them (values of $\left.r^{2}>0.997\right)$. The illustrative case of four representative phenols ( $p$-coumaric acid, protocatechuic acid, tyrosol, and $p$-hydroxyphenylacetic acid) are included in the Supplementary Information (Figure S2).
The quantum yield for reaction of DBO was previously defined by Nau in 1998 using ascorbic acid like antioxidant in aerated phosphate-buffer $(\mathrm{pH}=7)$. It was determined under conditions of quantitative $(\geq$ 95\%) quenching of singlet-excited DBO. For this purpose, DBO solutions (ca. $12 \mathrm{mM}$ ) with an absorbance of ca. 0.6 at the excitation wavelength of $351 \mathrm{~nm}$ were prepared, and ascorbic acid (30-40 mM) was added. Solutions of DBO in aerated water served as reference [quantum yield of decomposition $(\Phi \mathrm{r}) \approx$ $1 \%, \epsilon_{364}(\max )=48 \pm 3 \mathrm{M}^{-1} \mathrm{~cm}^{-1}, \epsilon_{351}(\max )=40 \pm$ $\left.2 \mathrm{M}^{-1} \mathrm{~cm}^{-1}\right] .{ }^{23}$

The bimolecular quenching rate constants $k_{q}\left(\mathrm{M}^{-1} \mathrm{~s}^{-1}\right)$ for singlet-excited DBO dissolved in water and in water/methanol was calculated (using eq. 4) for each phenolic compound (Table S1 in Supplementary Information). The $k_{q}$ values obtained for the samples dissolved in water/methanol are within the same order of magnitude $\left(10^{9} \mathrm{M}^{-1} \mathrm{~s}^{-1}\right)$, although slightly higher than the values of the same phenols dissolved in pure water. However, from a realistic and practical perspective (and because of the errors affecting the calculations of the $k_{q}$ values) the aforementioned differences can be considered negligible. Taking into account these observations and to facilitate the comparison with the DPPH assay results, the discussion of the DBO assay results will be limited as well to the water/methanol solvent ( $\mathrm{v} / \mathrm{v}$, 76/24).

\subsection{Temporal evolution of antioxidant reactivity of phenols}

To obtain a general understanding of the different interactions between the 15 phenols covered in this study and the DPPH free radical, and to know how these interactions evolve through time (from 0 to $90 \mathrm{~min}$ ), it was 
(a)

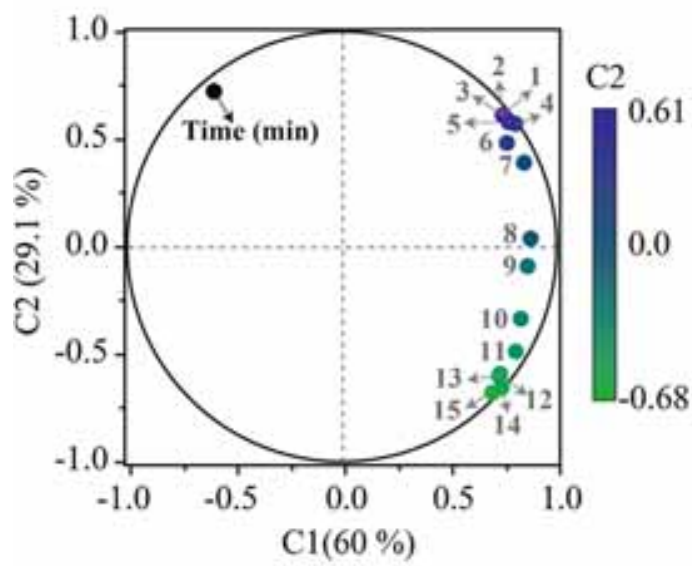

(b)

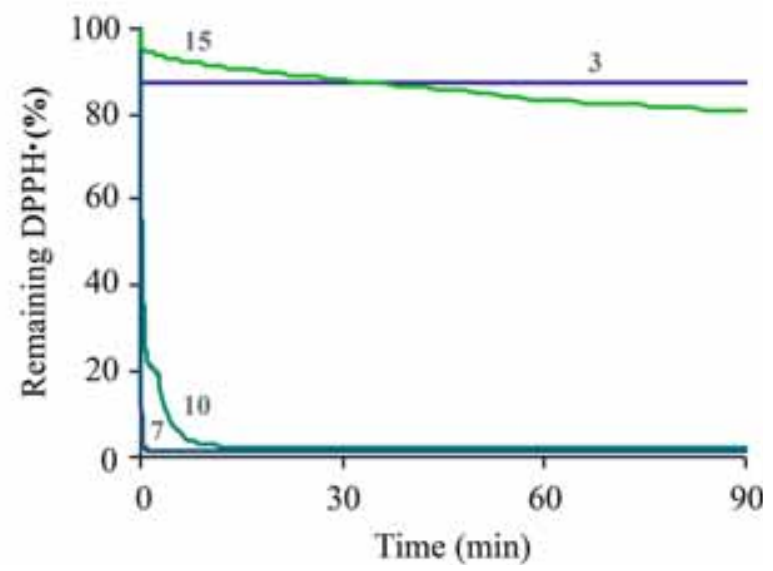

Figure 4. (a) Loadings plot obtained in the PCA study. Number designation is: (1) caffeic acid, (2) cinnamic acid, (3) p-hydroxybenzoic acid, (4) oleuropein, (5) sinapic acid, (6) hydroxytyrosol, (7) gallic acid, (8) protocatechuic acid, (9) syringic acid, (10) ferulic acid, (11) $p$-hydroxyphenylacetic acid, (12) tyrosol, (13) $p$-coumaric acid, (14) vanillic acid, and (15) $o$-coumaric acid. b) Time evolution of the remaining DPPH (\%) after addition of phenols. The following representative phenolic compounds were used to illustrate the study: (3) $p$-hydroxybenzoic acid, (7) gallic acid, (10) ferulic acid, and (15) $o$-coumaric acid in water/methanol $(\mathrm{v} / \mathrm{v}, 76 / 24)$.

decided to submit the results of the DPPH radical scavenging assay to a PCA statistical multivariate analysis. As can be observed in Figure 4a, 89.1\% of the original variance in the dataset can be explained using just 2 components (C1 60\% and C2 29.1\%).

The loadings for almost every variable in $\mathrm{C} 1$ are consistently higher than 0.7 (Table S2 in Supplementary Information) whereas the loadings obtained for $\mathrm{C} 2$ span a broad range (from -0.66 to 0.72 ). As expected, the most influential component $(\mathrm{C} 1)$ can be attributed to the global decrease shown by the DPPH free radical after reaction with every single phenol. This behavior is also visually and numerically offered in Figure $4 \mathrm{~b}$ and Table 1.

The interpretation of second component, although not as straight forward as the first one, has important information about the time dependent interaction between DPPH and phenols. At a first glance, it can be observed (Figure 4a) how phenols are progressively distributed between two extreme $\mathrm{C} 2$ loadings $(-0.68$ and 0.61$)$. All the phenols have in common induced an abrupt decrease (with different absolute values) in the DPPH concentration during the first second of interaction (Table 1 and Figure S2). However, each phenol has a particular residual signature after the starting period that can be fairly attributed to the variations in $\mathrm{C} 2$. For the sake of clarity, the following discussion will be focused on 4 representative compounds (Figure 4b), namely: $p$ hydroxybenzoic acid (3), gallic acid (7), ferulic acid (10) and $o$-coumaric acid (15). The almost symmet- rical position shown between $o$-coumaric (one of the boundary compounds in the $\mathrm{C} 2$ influence region) and time (Figure 4a), and the high inverse correlation coefficients observed between them $(-0.978$, Table S3 in Supplementary Information) can be explained by the constant decrease exhibited by the DPPH along the 90 minutes of the experiment (Figure 4b, line 15). On the other hand, the reaction between $p$-hydroxybenzoic acid and DPPH is completely stopped after the first second of interaction, generating a steady residual signature with no slope (Figure 4b). The almost null correlation coefficient between time and $p$-hydroxybenzoic acid $(-0.061$, Table S3 in Supplementary Information) also supports the time independent behavior of this residual signature. The evolution with time of the interactions between DPPH and gallic (7) and ferulic (10) acids are shown in Figure 4b to exemplify two intermediate behaviors within the $\mathrm{C} 2$ influence region.

The use of a single measurement at a defined time (e.g., after $30 \mathrm{~min}$ or $1 \mathrm{~h}$ of interaction between the DPPH radical and the antioxidant) is a common practice in the food industry to assess the antioxidant capacity of a specific sample or phenol. ${ }^{30,36}$ However, the results of the present study clearly show the diversity and complexity of the DPPH scavenging process and how critical it is to characterize the kinetics of the reactions involved. The previous generation of quantitative and qualitative information (i.e., Table 1 and Figure 4) about the kinetics of DPPH scavenging (or phenol antioxidant reactivity) 
Table 1. Remaining DPPH (\%) at increasing times ( $1 \mathrm{sec}, 10 \mathrm{sec}, 1 \mathrm{~min}, 5 \mathrm{~min}, 30 \mathrm{~min}, 90 \mathrm{~min})$ after each addition of phenol, and bimolecular rate constant for DBO fluorescence quenching $\left(k_{q}\right)$.

\begin{tabular}{|c|c|c|c|c|c|c|c|}
\hline & \multicolumn{6}{|c|}{ Remaining DPPH $(\%)$} & \multirow[t]{3}{*}{$k_{q} / 10^{9} \mathrm{M}^{-1} \mathrm{~s}^{-1[\mathrm{a}]}$} \\
\hline & \multicolumn{2}{|c|}{ Time (sec) } & \multicolumn{4}{|c|}{ Time (min) } & \\
\hline & 1 & 10 & $\mathrm{c}$ & 5 & 30 & 90 & \\
\hline$p$-Hydroxybenzoic acid & 87.4 & 87.4 & 87.4 & 87.4 & 87.4 & 87.4 & 0.6 \\
\hline Protocatechuic acid & 49.9 & 29.2 & 4.4 & 1.8 & 1.8 & 1.8 & 1.6 \\
\hline Gallic acid & 64.1 & 8.7 & 1.5 & 1.2 & 1.2 & 1.2 & 2.7 \\
\hline Vanillic acid & 94.3 & 93.2 & 87.8 & 84.5 & 74.0 & 66.5 & 1.0 \\
\hline Syringic acid & 73.1 & 36.2 & 7.9 & 1.6 & 1.2 & 1.2 & 1.6 \\
\hline Tyrosol & 94.3 & 90.4 & 62.4 & 46.0 & 34.8 & 21.9 & 1.1 \\
\hline Hydroxytyrosol & 76.3 & 9.0 & 8.3 & 8.3 & 8.3 & 8.3 & 1.3 \\
\hline$p$-Coumaric acid & 89.4 & 89.2 & 88.9 & 87.7 & 82.1 & 72.1 & 3.5 \\
\hline$o$-Coumaric acid & 96.1 & 96.1 & 95.2 & 93.2 & 88.4 & 81.1 & 7.9 \\
\hline Caffeic acid & 3.2 & 3.2 & 3.2 & 3.2 & 3.2 & 3.2 & - \\
\hline Cinnamic acid & 86.2 & 86.2 & 86.2 & 86.2 & 86.2 & 86.2 & - \\
\hline Ferulic acid & 74.7 & 53.3 & 22.2 & 6.7 & 2.2 & 2.2 & - \\
\hline Sinapic acid & 2.9 & 2.9 & 2.5 & 2.2 & 1.8 & 1.8 & - \\
\hline Oleuropein & 24.8 & 6.4 & 6.4 & 6.0 & 5.6 & 5.6 & 13.6 \\
\hline$p$-Hydroxyphenylacetic acid & 91.3 & 89.4 & 89.4 & 88.8 & 85.3 & 80.6 & 0.8 \\
\hline
\end{tabular}

[a]: Bimolecular rate constant for DBO fluorescence quenching in an aerated solvent mixture of $\mathrm{H}_{2} \mathrm{O} / \mathrm{MeOH}(\mathrm{v} / \mathrm{v}, 76 / 24) ; \tau_{0}$ $=75.3$ ns. Caffeic, cinnamic, ferulic and sinapic acids were insoluble in $\mathrm{H}_{2} \mathrm{O} / \mathrm{MeOH}$.

is crucial to design an exhaustive but at the same time efficient DPPH assay. More importantly, this knowledge provides important information about the selection of a specific phenolic compound as an antioxidant additive in food, pharmaceutical or cosmetic industries.

\subsection{Connections between antioxidant reactivity of phenols and molecular structure}

The previous section, while offering an idea of the diverse kinetic pathways shown by a selected group of phenolic compounds, naturally opens the discussion to investigate how the molecular structure of a phenol is related to its specific antioxidant reactivity. To tackle this question, the information obtained from the DPPH assay will be complemented with the results from the DBO assay.

It is well known that both the presence of a primary alcohol in the phenolic ring and the increment in the number of phenolic hydroxy radicals (-OH groups) increase antioxidant capacity of phenols. ${ }^{37,38}$ The presence of such specific structural features can be attributed to the different antioxidant reactivity observed for gallic, protocatechuic and $p$-hydroxybenzoic acids, which have in common the presence of a primary alcohol in the phenolic group but differ in the amount hydroxyl radicals (Figure 1). The following sequence for the remaining DPPH (\%), after 90 minutes of assay, can be observed (Table 1): gallic acid $(1.2 \%)<$ protocatechuic acid $(1.8 \%)<p$-hydroxybenzoic acid $(87.4 \%)$. Supporting this behavior, the $k_{q}$ values obtained in the DBO assay show the same trend with gallic acid $\left(2.7 \times 10^{9} \mathrm{M}^{-1} \mathrm{~s}^{-1}\right)$ displaying the highest antioxidant reactivity, protocatechuic acid $\left(1.6 \times 10^{9} \mathrm{M}^{-1} \mathrm{~s}^{-1}\right)$ an intermediate one and $p$-hydroxybenzoic acid $(0.6 \times$ $10^{9} \mathrm{M}^{-1} \mathrm{~s}^{-1}$ ) the lowest one. Thus, the differences in the antioxidant reactivity of these three phenols can be attributed to the presence of three, two and one hydroxyl radicals in the structure of gallic acid, protocatechuic acid and $p$-hydroxybenzoic acid, respectively.

Another structural feature that may control the antioxidant reactivity of phenolic compounds is the presence of methoxy groups, because they are electron-donating groups that help stabilizing the produced phenoxy radicals (reaction 1). In this regard, caffeic and sinapic acids (incorporating two hydroxyl groups and one hydroxyl plus two methoxy groups, respectively) showed an almost equal and instant antioxidant reaction with DPPH (Table 1). This observation suggests that the electronic effects of one hydroxyl group and two methoxy groups are comparable. This feature has also been observed for protocatechuic acid and syringic acids, with two hydroxyl groups and one hydroxyl plus two methoxy groups, respectively. In accordance with this reasoning, the antioxidant reactivity of ferulic acid is 
lower than that of caffeic acid and sinapic acid because the former contains only one hydroxyl and one methoxy group (Table 1 and Figure S3 in Supporting Information).

A similar process (well-stabilized phenoxy radicals as a consequence of an increase in the electron-donating activity) may be attributed to the presence of an alkyl chain. ${ }^{39}$ This structural feature (as well as the common presence of only one hydroxyl group) can be responsible of the low antioxidant reactivity showed by $p$-coumaric, $p$-hydroxyphenylacetic and $p$-hydroxybenzoic acids.

According to the literature report, a hydroxyl group in an ortho position induces higher antioxidant reactivity. ${ }^{10}$ In this regard, when the $k_{q}$ values of $o$-coumaric and $p$-coumaric acids are compared (Table 1), it can be seen how $o$-coumaric acid induces a higher antioxidant reactivity $\left(7.9 \times 10^{9} \mathrm{M}^{-1} \mathrm{~s}^{-1}\right)$ than $p$-coumaric acid $\left(3.5 \times 10^{9} \mathrm{M}^{-1} \mathrm{~s}^{-1}\right)$.

Additionally, it is well-known that the presence of an alkenyl chain stabilizes the formed phenoxy radicals. ${ }^{11,37}$ In accordance with that, caffeic $\left(\mathrm{R}_{2}, \mathrm{R}_{3}=\right.$ $\mathrm{OH})$, ferulic $\left(\mathrm{R}_{2}=\mathrm{CH}_{3} \mathrm{O} ; \mathrm{R}_{3}=\mathrm{OH}\right)$ and sinapic $\left(\mathrm{R}_{2}\right.$, $\mathrm{R}_{4}=\mathrm{CH}_{3} \mathrm{O} ; \mathrm{R}_{3}=\mathrm{OH}$ ) acids showed the expected lower values of remaining DPPH when compared to protocatechuic $\left(\mathrm{R}_{2}, \mathrm{R}_{3}=\mathrm{OH}\right)$, vanillic $\left(\mathrm{R}_{3}=\mathrm{CH}_{3} \mathrm{O}\right.$; $\left.\mathrm{R}_{2}=\mathrm{OH}\right)$ and syringic $\left(\mathrm{R}_{1}, \mathrm{R}_{3}=\mathrm{CH}_{3} \mathrm{O} ; \mathrm{R}_{2}=\mathrm{OH}\right)$ acids, respectively. $k_{q}$ values of Caffeic, ferulic and sinapic acids were not obtained because these phenols were insoluble in water/methanol mixture.

Both approaches showed a clear correspondence between the structure and the antioxidant reactivity of each of the studied phenolic compounds. This interesting resemblance supports the use of the more novel DBO method as a complementary method to measure the antioxidant reactivity of phenolic compounds.

\subsection{Conclusions}

Two methodologies (fluorescence probe, $n, \pi^{*}$ singlet excited state of 2,3-diazabicyclo[2.2.2]oct-2-ene (DBO) and the free radical 2,2-diphenyl-1-picrylhydrazyl $\left.\left(\mathrm{DPPH}^{-}\right)\right)$were tested in order to determine the antioxidant reactivity of the most typical phenolic compounds present in virgin olive oil. The study showed that both methodologies work well by providing valuable information about the antioxidant reactivity of the compound. By knowing the role played by specific structural features in the antioxidant reactivity of phenols, new compounds could be designed with desired antioxidant reactivity or some other compounds could be functionalized for a required task. The information provided in this study, as well as in the many studies on the antioxidant reactivity vs molecular structure of phenols, will help to build the foundations of food, pharmaceutical and cosmetic products functionalized with antioxidants properties.

\section{Supplementary Information (SI)}

All additional information pertaining to final absorbance of DPPH obtained for each phenolic compound in methanol and in water/methanol mixture (v/v,76/24) (Figure S1), kinetic Stern-Volmer plots (Figure S2), remaining DPPH (\%) achieved when $1 \mathrm{~mL}$ of each phenolic compound dissolved in water/methanol (v/v, 76/24) mixture was added to $2 \mathrm{~mL}$ of DPPH dissolved in methanol (Figure S3), bimolecular rate constant for DBO fluorescence quenching $\left(k_{q}\right)$ obtained for the studied phenolic compounds (Table S1), loadings matrix for the 2 components combining the 16 variables studied (Table S2) and Spearman correlation matrix of the 16 variables analyzed (Table S3) are given in the Supporting Information available at www.ias.ac.in/chemsci.

\section{Acknowledgements}

We are grateful to Prof. W. M. Naufrom Jaccobs University of Bremen (Germany) for kindly providing the DBO fluorophore used in this study, and to Prof. J. L. Espartero from University of Seville (Spain) for kindly providing the hydroxytyrosol. We are also indebted to Prof. M. A. Caraballo and Prof. P. Richter from University of Chile (Chile) and Prof. U. Pischel from University of Huelva (Spain) for helpful comments and discussions. This study was funded by the Environmental Council of the Andalusia Regional Government through project P10 FQM 6185 and by the Operative Program of Transborderline Cooperation Spain-Portugal through project 0434_I2TEP_5_E OLITRACE and by FONDECYT (Postdoctoral Grant 3150059).

\section{References}

1. Orzechowski A, Ostaszewski P, Jank M and Berwird S J 2002 Bioactive substances of plant origin in food-impact on genomics Reprod. Nutr. Dev. 42461

2. Saura-Calixto F and Goñi I 2006 Antioxidant capacity of the Spanish Mediterranean diet Food Chem. 94442

3. Bendini A, Cerretani L, Carrasco-Pancorbo A, GómezCaravaca A M, Segura-Carretero A, FernándezGutiérrez A and Lercker G 2007 Phenolic molecules in virgin olive oils: a survey of theirsensory properties, health effects, antioxidant activity and analytical methods. An overview of the last decade Molecules 121679

4. Beer de D, Joubert E, Gelderblom W C A and Manley M 2002 Phenolic compounds: a review of their possible role as in vivo antioxidants of wine $S$. Afr. J. Enol. Vitic. 23 48

5. Perona J S, Cabello-Moruno R and Ruíz-Gutierrez V 2006 The role of virgin olive oil components in the modulation of endothelial function J. Nutr. Biochem. 17429 
6. Belay A and Gholap A V 2009 Characterization and determination of chlorogenic acids (CGA) in coffee beans by UV-Vis spectroscopy AJPAC 11234

7. Trichopoulou A and Lagiou P 1997 Healthy traditional Mediterranean diet: an expression of culture, history, and lifestyle Nutr. Rev. 55383

8. Camuesco D, Comalada M, Rodriguez-Cabezas M E, Nieto A, Lorente M D, Concha A, Zarzuelo A and Galvez J 2004 The intestinal anti-inflammatory effect of quercitrin is associated with an inhibition in iNOS expression Br. J. Pharmacol. 143908

9. Yu T, Lee Y J, Jang H J, Kim A R, Hong S, Kim T W, Kim M Y, Lee J, Lee Y G and Cho J Y 2011 Antiinflammatory activity of Sorbuscommixta water extract and its molecular inhibitory mechanism J. Ethnopharmacol. 134493

10. Lucarini M and Pedulli G F 1994 Bond dissociation enthalpy of $\alpha$-tocopherol and other phenolic antioxidants J. Org. Chem. 595063

11. Nenadis N, Boyle S, Bakalbassis E G and Tsimidou M 2003 An experimental approach to structure-activity relationships of caffeic and dihydrocaffeic acids and related monophenols JAOCS $\mathbf{8 0} 451$

12. Chandraker K, Vaishanav S K, Nagwanshi R and Satnami M L 2015 Radical Scavenging Efficacy of Thiol Capped Silver Nanoparticles J. Chem. Sci. 1272183

13. Koprivnjak O, Škevin D, Valić S, Majetić V, Petričević S and Ljubenkov I 2008 The antioxidant capacity and oxidative stability of virgin olive oil enriched with phospholipids Food Chem. 111121

14. Yapati H, Devineni S R, Chirumamilla S and Kalluru S 2016 Synthesis, characterization and studies on antioxidant and molecular docking of metal complexes of 1-(benzo[d]thiazol-2-yl)thiourea J. Chem. Sci. 128 43

15. Poerio A, Bendini A, Cerretani L, Bonoli-Carbognin M and Lercker G 2008 Effect of olive fruit freezing on oxidative stability of virgin olive oil Eur. J. Lipid Sci. Technol. 110368

16. Bendini A, Cerretani L, Vecchi S, Carrasco-Pancorbo A and Lercker G 2006Protective Effects of Extra Virgin Olive Oil Phenolics on Oxidative Stability in the Presence or Absence of Copper Ions J. Agric. Food Chem. 54 4880

17. Ou B, Huang D, Hampsch-Woodill M, Flanagan J A and Deemer E K 2002 Analysis of Antioxidant Activities of Common Vegetables Employing Oxygen Radical Absorbance Capacity (ORAC) and Ferric Reducing Antioxidant Power (FRAP) Assays: A Comparative Study J. Agric. Food Chem. 503122

18. Einbond L S, Reynertsona K A, Xiao-Dong L, Basileb M J and Kennelly E J 2004 Anthocyanin antioxidants from edible fruits Food Chem. 8423

19. Pischel U, Patra D, Koner A L and Nau W M 2006 Investigation of polar and stereoelectronic effects on pure excited-state hydrogen atom abstractions from phenols and alkylbenzenes J. Photochem. Photobiol. 82310

20. Anbazhagan V, Kandavelu V, Kathiravan A and Renganathan R 2008 Investigation on the fluorescence quenching of 2,3-diazabicyclo[2.2.2] oct-2-ene (DBO) by certain estrogens and catechols J. Photochem. Photobiol. A: Chem. 193204
21. Anbazhagan V, Kalaiselvan A, Jaccob M, Venuvanalingam $\mathrm{P}$ and Renganathan R 2008 Investigations on the fluorescence quenching of 2,3diazabicyclo[2.2.2] oct-2-ene by certain flavonoids $J$. Photochem. Photobiol. B: Biol. 91143

22. Anbazhagan V and Renganathan R 2009 Investigation of the fluorescence quenching of 2,3diazabicyclo[2.2.2] oct-2-ene(DBO) by certain substituted uracils J. Lumin. 129382

23. Nau W M 1998 A fluorescent probe for antioxidants JACS 12012614

24. Žilić D, Pajić D, Jurić M,Molčanov K, Rakvin B, Planinić P and Zadro K 2010 Single crystals of DPPH grown from diethyl ether and carbon disulfide solutions - crystal structures, IR, EPR and magnetization studies J. Magn. Res. 20734

25. Zhang $\mathrm{Z}$ L, Chen J, Xu Q, Rao C and Qiao C 2011 Efficient Synthesis of Hydroxytyrosol from 3,4Dihydroxybenzaldehyde Synth. Commun. 42794

26. Askani R 1965 Zur Reaction von Cyclohexadien-(1.3) mitAzodicarbonsäure-diäthylester Chem. Beri. 982551

27. Jolliffe T 2002 In Principal Component Analysis (New York: Springer Verlag)

28. Brand-Williams W, Cuvelier M E and Berset C1995 Use of a free radical method to evaluate antioxidant activity LWT-Food Sci.Tecnol. 2825

29. Miliauskas G, Venskutonis P R and Van Beek T A 2004 Screening of radical scavenging activity of some medicinal and aromatic plant extracts Food Chem. 85231

30. Dudonné S, Vitrac X, Coutière P, Woillez M and Mérillon J M 2009 Comparative Study of Antioxidant Properties and Total Phenolic Content of 30 Plant Extracts of Industrial Interest Using DPPH, ABTS, FRAP, SOD, and ORAC Assays J. Agric. Food Chem. 571768

31. Pizarro M L, Becerra M, Sayago A, Beltrán M and Beltrán R 2013 Comparison of Different Extraction Methods to Determine Phenolic Compounds in Virgin Olive Oil Food Anal. Method 6123

32. Becerra-Herrera M, Sánchez-Astudillo M, Beltrán R and Sayago A 2014 Determination of phenolic compounds in olive oil: New method based on liquid liquid micro extraction and ultra-high performance liquid chromatography-triple-quadrupole mass spectrometry LWT-Food Sci. Tecnol. 5749

33. López-Giraldo L J, Laguerre M, Lecomte J, FigueroaEspinoza M C, Baréa B, Weiss J, Decker E A and Villeneuve P 2009 Kinetic and Stoichiometry of the Reaction of Chlorogenic Acid and Its Alkyl Esters against the DPPH Radical J. Agric. Food Chem. 57863

34. Nau W M, Greiner G, Rau H, Wall J, Olivucci M and Scaiano J C 1999 Fluorescence of 2,3diazabicyclo[2.2.2] oct-2-ene revisited: Solvent-induced quenching of the $\mathrm{n}, \pi^{*}$-excited state by an aborted hydrogen atom transfer J. Phys. Chem. A 1031579

35. Foti M C, Daquino C, DiLabio G A and Ingold K U 2011 Kinetics of the oxidation of quercetin by 2,2-diphenyl1-picrylhydrazyl (dpph•) Org. Lett. 134826

36. Ajitha M J, Mohanlal S, Suresh $\mathrm{C} \mathrm{H}$ and Jayalekshmy A 2012 DPPH Radical Scavenging Activity of Tricin and Its Conjugates Isolated from "Njavara" Rice Bran: A Density Functional Theory Study J. Agric. Food Chem. 603693 
37. Rice-Evans A C, Miller N J and Paganga G 1996 Structure-antioxidant activity relationship of flavonoids and phenolic acids Free Radical Biol. Med. 20933

38. Campos A M, Duran N, Lopez-Alarcón C and Lissi E 2012 Kinetic and stoichiometric evaluation of free radicals scavengers activities based on diphenyl- picrylhydrazyyl (DPPH) consumption J. Chil. Chem. Soc. $\mathbf{5 7} 1381$

39. Torres de Pinedo A, Peñalver P and Morales J C 2007 Synthesis and evaluation of new phenolic-based antioxidants: Structure-activity relationship Food Chem. 103 55 